\title{
Exercise in Anorexia Nervosa: Complexity of Pathology and Health
} Trevor Archer ${ }^{1,2^{*}}$ and Danilo Garcia ${ }^{2-5}$

${ }^{1}$ University of Gothenburg, Department of Psychology, Box 500, 40530 Gothenburg, Sweden

${ }^{2}$ Network for Empowerment and Well-Being, Sweden

${ }^{3}$ Blekinge Centre of Competence, Karlskrona, Sweden

${ }^{4}$ Departments of Psychiatry and Genetics, Sansone Center for Well-being, Washington University School of Medicine in St. Louis, Missouri, USA

5 Institute of Neuroscience and Physiology, Centre for Ethics, Law and Mental Health (CELAM), University of Gothenburg, Gothenburg, Sweden

\section{Editorial}

Anorexia nervosa (AN) condition presents a serious and potentially life-threatening eating disorder characterized by episodes of self-starvation and excessive weight loss that may be accompanied by excessive exercise [1]. The notion that the condition offers a dysfunctional system of individuals' self-evaluation of self-worth, where self-worth as determined from perceived body shape and weight and the degree to which they consider themselves to be in control of their own shape and weight. The control need and necessity for specific body shape and weight appears to drive the pathologic dietary restraint and the consequent operationalization of these tendencies into various behavioural expressions and dietary rules that may either lead to success, e.g. outcome weight loss, or fail in implementation, e.g. resulting in subjective or objective binge eating episodes for example, often induced by the failure of restriction to dietary rules [2]. These notions support the essential over-evaluation of weight and shape and that this cognitive mind-set in its turn influences the attempts at eating restraint [3]. It has been observed also that low individual self-esteem exerts a general influence on over-evaluation of weight and shape [4]. Since low self-esteem in its turn has been shown to be associated with higher negative affect as measured with Positive Affect and Negative Affect Schedule [5,6], it appears conceivable that individuals afflicted with the psychopathology condition experience less positive and more negative affect, as a consequence of low self-esteem. Physical exercise influences cognitive, emotional, learning and neurophysiological domains, both directly and indirect, thereby rendering it essential that this noninvasive, non-pharmacological intervention ought to form a part of children's and adolescents' long-term health programs $[7,8]$.

Introducing exercise regimes has been found to be beneficial to the health and well-being of individuals, both under laboratory and clinical conditions and has been demonstrated using a variety of health guidelines and biomarkers [9]. Physical, bodily, activities enhancing or maintaining physical fitness mobilize the engagement of regular and frequent exercise thereby ensuring physical fitness to the exclusion of agents associated with health problems, e.g. pro-inflammatory cytokines and cortisol. Taking into account, the large proportion of individuals with more-or-less sedentary preoccupations, physical exercise presents uniquely one of the most potent health-promoting lifestyles available by rendering positive outcomes for both neurologic and psychiatric conditions [10-15]. It is beneficial also within domains defined by neurocognitive capacity, quality-of-life, postural and motor efficacy and affective status [16-18]. Generally, exercise has been defined as a planned, structured physical activity with the purpose of improving one or more aspects of physical fitness and functional capacity [19]. Health status may be estimated by the level of functional or metabolic efficiency of living organisms; in this context, the type, duration and extent of physical exercise may be an essential issue in determining its interventional status. For example, the influence of physical activity upon subjective well-being was studied through an analysis of individuals' participation intensity and duration of sports activities [20].
The issue or whether or not physical exercise serves patients presenting AN optimally remains a markedly complicated issue since the prevalence of problematic exercise fluctuates to a great extent from 5\% to $54 \%$, depending upon the number of criteria applied for its definition [21]. The enduringly, but disturbingly, high level of variation depicting the physical exercise/activity profiles of AN patients that is attributed to the, either low or very high, patterns of these behaviors presents a highly relevant issue in AN [22]. It has been shown that there are differences expressed between those parents with and those without core eatingdisorder psychopathology; those parents presenting eating-disorder psychopathology reported greater perceived feeding responsibility, stronger concerns about their children's weight management and spent more time monitoring the children's intake behavior than those parents not presenting eating-disorder characteristics [23]. The notion concerning the conceptualization and measurement of pathological exercise in association with eating disorder, not least $\mathrm{AN}$, is not novel, not least in its connotations with perfectionism and compulsive behaviour [24]. In a sample of 1,497 adult ( 608 men, 885 women, 4 other) individuals, it was found that those linked to physical exercise and an accompanying eating disorder, i.e. secondary PE, scored higher than those with physical exercise and no eating disorder symptoms, i.e. primary ED, on the compulsive exercise test and the Dimensional Obsessive-Compulsive Scale while correlations between Eating Disorders Examination Questionnaire and physical exercise indicated that scores were at higher levels among women than among men [25]. Furthermore, sixty female adolescent inpatients presenting AN in a self-report measure indicated that compulsive exercise was driven by avoidance of negative affect and was associated with more severe psychological features.

Several core themes have been identified to clarify the utility of exercise in AN; these include (i) the employment of a team of relevant experts, (ii) the monitoring patients' medical status, (iii) the development of screens for exercise-related psychopathology, (iv) the creation and compliance with a written contract of how therapeutic exercise will be applied, (v) the inclusion of a psychoeducational component, (vi) focusing upon positive reinforcement, (vii) the creation of a graded exercise program, beginning with mild-intensity exercise schedules, (viii) constructing tailor-made modes of exercise to the needs of the AN patient, (ix) the inclusion of a nutritional component, and, finally, $(\mathrm{x})$ the essential debriefing procedure for

*Corresponding author: Archer T, Department of Psychology, University of Gothenburg, Box 500, S-405 30 Gothenburg, Sweden, Tel: +46 317864694 : E-mail: trevor.archer@psy.gu.se

Received: July 20, 2016; Accepted: July 21, 2016; Published: July 28, 2016

Citation: Archer T, Garcia D (2016) Exercise in Anorexia Nervosa: Complexity of Pathology and Health. Clin Exp Psychol 2: e107. doi:10.4172/2471-2701.1000e107

Copyright: (c) 2016 Archer T, et al. This is an open-access article distributed under the terms of the Creative Commons Attribution License, which permits unrestricted use, distribution, and reproduction in any medium, provided the original author and source are credited. 
Citation: Archer T, Garcia D (2016) Exercise in Anorexia Nervosa: Complexity of Pathology and Health. Clin Exp Psychol 2: e107. doi:10.4172/24712701.1000e107

patients following the exercise sessions. It has obtained two 'overarching' themes among AN women: (i) the 'paradoxical functions of exercise' and (ii) the 'diverging experiences of exercise'; by the former despite severe extent of underweightedness and exhaustion endurance combined with a distinctive awareness of the associated negative health effects, these participants maintained a continuous cycle of rigorous and excessive exercise that exhausted extensive amounts of time and energy. By the latter, exercise schedules exerted a negative control and interference with the participants' involvement in their surrounding social context, where their references to exercise divulged the choice of words to be distinguished by efforts to 'down-play' the exerciseextent immersion. These issues were complicated by the position that control and ambivalence regarding disorder treatment and recovery presented potential triggers for the participants' adherence to an exercise lifestyle. Applying acute phenylalanine/tyrosine depletion to decrease temporarily dopamine synthesis and transmission in AN patients and healthy controls, it was observed that patients recovering from AN were more motivated to exercise than the healthy controls in spite of the situation that in both groups, exercise activity was more reinforcing than inactivity. The results imply that reduced dopamine did not lessen the motivation to exercise in patients recovered from AN, but contrastingly, did so in the healthy controls. The authors have proposed that in patients afflicted with AN, the drive to exercise develops into a compulsion that is to a great extent independent of dopamine-mediated reward processes and develops a dependency on cortico-striatal neurocircuitry that regulates the automated, habit- or compulsive-like behaviors expressed in the disorder. Nevertheless these considerations may be vital since after the restoration of healthy weight levels in AN, and in particular by the time spent in standing postures, physical exercise pathology recurrence may result in weight loss during the year following hospitalization [25].

In conclusion, exercise benefits, confirmed for a large number of neuropsychiatric and neurologic conditions, seem not to be unrestrictedly the intervention of choice for AN although the healthenhancing and well-being-endowering influences remain undeniable.

\section{References}

1. Moradi S, Nima AA, Rapp Ricciardi M, Archer T, Garcia D (2014) Exercise character strengths, well-being, and learning climate in the prediction of performance over a 6-month period at a call center. Front Psychol 5: 497.

2. Archer T, Kostrzewa RM (2016) Exercise and Nutritional Benefits in PD: Rodent Models and Clinical Settings. Curr Top Behav Neurosci 29: 333-51.

3. Archer T, Kostrzewa RM (2015) Physical Exercise Alleviates Health Defects, Symptoms, and Biomarkers in Schizophrenia Spectrum Disorder. Neurotox Res 28: 268-80.

4. Archer $T$ (2014) Health benefits of physical exercise for children and adolescents. J Novel Physiother 4: 203.

5. Garcia D, Archer T (2014) Positive affect and age as predictors of exercise compliance. PeerJ 2: e694.

6. Garcia D, Jimmefors A, Mousavi F, Adrianson L, Rosenberg P, et al. (2015b) Self-regulatory mode (locomotion and assessment), well-being (subjective and psychological), and exercise behavior (frequency and intensity) in relation to high school pupils' academic achievement. Peer J 3: e847.
7. Noetel M, Miskovic-Wheatley J, Crosby RD, Hay P, Madden S, et al. (2016) A clinical profile of compulsive exercise in adolescent inpatients with anorexia nervosa. J Eat Disord 4: 1.

8. O'Hara CB, Keyes A, Renwick B, Leyton M, Campbell IC, et al. (2016) The Effects of Acute Dopamine Precursor Depletion on the Reinforcing Value of Exercise inAnorexia Nervosa. PLoS One 11: e0145894.

9. Stoeber J, Madigan DJ, Damian LE, Esposito RM, Lombardo C (2016) Perfectionism and eating disorder symptoms in female university students: the central role of perfectionistic self-presentation. Eat Weight Disord.

10. Wicker P, Frick B (2015) The relationship between intensity and duration of physical activity and subjective well-being. Eur J Public Health.

11. Goodwin H, HaycraftE, Meyer C (2016) Disordered Eating, Compulsive Exercise and Sport Participation in a UK Adolescent Sample. Eur Eat Disord Rev 24 304-9.

12. Holland G, Tiggemann M (2016) "Strong beats skinny every time": Disordered eating and compulsive exercise in women who post fitspiration on Instagram. Int J Eat Disord.

13. Archer T, Garcia D (2014) Physical exercise influences academic performance and well-being in children and adolescents. I JSchool Cogn Psychol 1: e102.

14. Cunningham HE, Pearman S, Brewerton TD (2016) Conceptualizing primary and secondary pathological exercise using available measures of excessive exercise. Int J Eat Disord.

15. Archer T (2012) Influence of physical exercise on traumatic brain injury deficits: scaffolding effect. Neurotox Res 21: 418-34.

16. Cook BJ, Wonderlich SA, Mitchell JE, Thompson R, Sherman R, et al. (2016) Exercise in Eating Disorders Treatment: Systematic Review and Proposal of Guidelines. Med Sci Sports Exerc 48: 1408-14.

17. Kolnes LJ (2016) 'Feelings stronger than reason': conflicting experiences of exercise in women with anorexia nervosa. J Eat Disord 4: 6.

18. Lydecker JA, Grilo CM (2016) Fathers and mothers with eating-disorder psychopathology: Associations with child eating-disorder behaviors. J Psychosom Res 86: 63-9.

19. Gianini LM, Klein DA, Call C, Walsh BT, Wang Y, et al. (2016) Physical activity and post-treatment weight trajectory in anorexia nervosa. Int J Eat Disord 49: 482-9.

20. Rizk M, Lalanne C, Berthoz S, Kern L, Godart N (2015) Problematic Exercise in Anorexia Nervosa: Testing Potential Risk Factors against Different Definitions. PLoS One 10: e0143352.

21. Danielsen M, Rø $\varnothing$, Romild U, Bjørnelv S (2016) Impact of female adult eating disorder inpatients' attitudes to compulsive exercise on outcome at discharge and follow-up. J Eat Disord 4: 7 .

22. Sauchelli S, Arcelus J, Sánchez I, Riesco N, Jiménez-Murcia S, et al. (2015) Physical activity in anorexia nervosa: How relevant is it to therapy response? Eur Psychiatry 30: 924-31.

23. Archer T, Garcia D (2015) Exercise and dietary restriction for promotion of neurohealth benefits. Health 7: 136-152.

24. Archer T, Josefsson T, Lindwall M (2014) Effects of physical exercise on depressive symptoms and biomarkers in depression. CNS Neurol Disord Drug Targets 13: 1640-53.

25. Garcia D, MacDonald S, Archer T (2015a) Two different approaches to the affective profiles model: median splits (variable-oriented) and cluster analysis (person-oriented). PeerJ 3: e1380. 